\title{
Impacts of caring for a child with the CDKL5 disorder on parental wellbeing and family quality of life
}

\author{
Yuka Mori ${ }^{1}$, Jenny Downs ${ }^{1,2}$, Kingsley Wong ${ }^{1}$, Barbara Anderson ${ }^{1}$, Amy Epstein $^{1}$ and Helen Leonard ${ }^{1 *}$ (D)
}

\begin{abstract}
Background: Although research in this area remains sparse, raising a child with some genetic disorders has been shown to adversely impact maternal health and family quality of life. The aim of this study was to investigate such impacts in families with a child with the CDKL5 disorder, a newly recognised genetic disorder causing severe neurodevelopmental impairments and refractory epilepsy.
\end{abstract}

Methods: Data were sourced from the International CDKL5 Disorder Database to which 192 families with a child with a pathogenic CDKL5 mutation had provided data by January 2016. The Short Form 12 Health Survey Version 2, yielding a Physical Component Summary and a Mental Component Summary score, was used to measure primary caregiver's wellbeing. The Beach Center Family Quality of Life Scale was used to measure family quality of life. Linear regression analyses were used to investigate relationships between child and family factors and the various subscale scores.

Results: The median (range) age of the primary caregivers was 37.0 (24.6-63.7) years and of the children was 5.2 (0.2-34.1) years. The mean (SD) physical and mental component scores were 53.7 (8.6) and 41.9 (11.6), respectively. In mothers aged 25-54 years the mean mental but not the physical component score was lower than population norms. After covariate adjustment, caregivers with a tube-fed child had lower mean physical but higher mean mental component scores than those whose child fed orally (coefficient $=-4.80$ and $6.79 ; p=0.009$ and 0.012 , respectively). Child sleep disturbances and financial hardship were negatively associated with the mental component score. The mean (SD) Beach Center Family Quality of Life score was 4.06 (0.66) and those who had used respite services had lower scores than those who had not across the subscales.

Conclusions: Emotional wellbeing was considerably impaired in this caregiver population, and was particularly associated with increased severity of child sleep problems and family financial difficulties. Family quality of life was generally rated lowest in those using respite care extensively, suggesting that these families may be more burdened by daily caregiving.

Keywords: Parental wellbeing, Family quality of life, CDKL5 disorder, Genetic disorder, Rare disorder

\footnotetext{
*Correspondence: Helen.Leonard@telethonkids.org.au

${ }^{1}$ Telethon Kids Institute, The University of Western Australia, 100 Roberts

Road, Subiaco, 6008 Perth, Western Australia

Full list of author information is available at the end of the article
} 


\section{Background}

The CDKL5 disorder is a recently identified genetic disorder caused by mutations in the X-linked cyclindependent kinase-like 5 (CDKL5) gene [1]. These mutations were originally identified in children or adults who had been clinically diagnosed with epileptic encephalopathy [2] or the early-onset seizure variant of Rett syndrome (RTT) [3]. However, there has been recent evidence demonstrating some differences in clinical features when compared with typical RTT [4-6]. Epilepsy is almost universal and generally occurs in early infancy with a median age of six weeks ranging from one week to 1.5 years [4, 7] and thus symptoms of this disorder appear very early in life. Sleep difficulties appear to be more common in the CDKL5 disorder than in RTT $[4,6]$. Genotype-phenotype relationships have been difficult to study because of the paucity of recurrent mutations and the generally small case series. Our recent study $(n=127)$ which examined early development in this disorder categorised mutation types into four groups according to the effect on protein function and found that compared with those with no functional protein, those with a truncating mutation after amino acid (aa) 781 were more likely to acquire motor and communication skills [8].

Growing evidence suggests that raising a child with a disability greatly impacts the welfare of the primary caregiver, particularly, the mother. Although there is an increasing number of studies examining maternal health $[9,10]$, research specifically targeting families caring for a child with a genetic disorder remains relatively scarce. While impaired mental health has been reported for those with a child with Down syndrome [11] and RTT [12, 13], and increased stress for those with a child with Prader-Willi syndrome [14], there are no data on the health of parents caring for a child with the CDKL5 disorder.

The concept of family quality of life for those with a child with a developmental disability has developed along with understanding of the crucial role that family plays in raising a child with a disability [15]. Identifying specific challenges for the family is critical to providing an inclusive and multidisciplinary healthcare strategy [16]. The burden of care may impact more greatly on families with a child who have more severe neurodevelopmental impairments accompanied with complex comorbidities and this is typical in many rare disorders. Often, the child's diagnosis and access to syndromespecific family support can be considerably delayed, adversely affecting parental wellbeing [17] and possibly also family quality of life.

The aim of this study is to examine primary caregivers' wellbeing and family quality of life among families with a child living with the CDKL5 disorder, and determine the relationships with a range of factors from child's characteristics through family circumstances to availability of public resources. This study is the first investigation into family life for those raising a child with the CDKL5 disorder.

\section{Methods \\ Data collection}

Prior to 2012 the international Rett syndrome database, InterRett, set up in 2002, and mainly collecting data using electronic data submission [4, 18], also included cases with the CDKL5 disorder. In 2012 the International CDKL5 Disorder Database (ICDD) was established by recontacting those InterRett families in whose child a CDKL5 gene mutation had been identified, and by recruiting new cases in collaboration with the International Foundation for CDKL5 Research (IFCR). Following registration on the IFCR webpage, an ICDD staff member contacts the family, explains the study and provides instructions on how they can complete the questionnaire and provide their genetic test results. The questionnaire comprises two primary components and an epilepsy diary. The first part includes sections that provide a comprehensive picture of the child's clinical condition from birth to present: perinatal details; primary concerns and diagnosis; developmental milestones; regression; current functional ability, including gross motor, communication and feeding; past and current seizure frequency and medication; sleep difficulties; emotional and social development; stereotypic hand movements; gastrointestinal symptoms; skeletal and muscular health; other comorbidities; hospitalisations; drug treatments; stage of puberty; current body measurements; equipment and intervention use; day care and educational supports; and utilisation of respite care and financial aids. Questions were also asked about the specific forms of respite care used, where formal respite included services provided by public or private organisations and informal respite referred to any service offered by other family members, friends and neighbours. The second part includes sections regarding family demographics, family wellbeing using the Beach Center Family Quality of Life (BCFQOL) Scale, and parental wellbeing using the Short Form 12 Health Survey Version 2 (SF-12v2).

For the current study, data were collected between September 2012 and January 2016. Of those with a confirmed pathogenic mutation, 192 of 209 provided questionnaire data. One hundred and fifty-eight families (82.2\% of 192) were included in the parental health analysis excluding 34 of those with three or more missing items. Of those, 141 (89.2\%) questionnaires were completed by the biological mother, 15 (9.5\%) by the biological father, and one $(0.6 \%)$ each by a foster mother 
and a grandparent. In the family quality of life analysis, 149 families with fewer than three missing items (77.6\% of 192) were included. The questionnaires were filled out by 131 (87.9\%) biological mothers, 16 (10.7\%) biological fathers, one foster mother $(0.7 \%)$ and one grandparent $(0.7 \%)$.

\section{Measurements}

\section{Child's mutation group}

There was considerable variability in mutations in the CDKL5 gene with 149 unique and only 18 recurrent mutations. Hence, every mutation was categorised into one of five groups according to the subsequent protein function: No functional protein; Missense/in-frame mutations within kinase domain; Truncations between aa172 and aa781; Truncations after aa781; and mutations that could not be grouped [8].

\section{Child's functional abilities}

For those aged 1.5 years or over, abilities to sit on the floor for ten seconds and to walk ten steps were categorised as needing no assistance, needing some support or unable. Also in those aged 1.5 years or over, ability to communicate was categorised as using no or simple communication methods (eg, body language and facial expressions), complex gestures and vocalisation (eg, selects or rejects a photo of object, points at an object or returns an unwanted item) or using sign or spoken language (eg, single words and sentences). Children or adults who were totally dependent on enteral feeding were excluded from analyses relating to dietary concerns.

\section{Child's sleep}

The Sleep Disturbance Scale for Children (SDSC) has been validated in children with disability as well as healthy children [19]. It contains 26 items rated on a five-point Likert scale [19]. Four items were not included in the questionnaire as they were not appropriate to this population (e.g. the child sleep walks). The average score of the remaining 22 items was calculated with a possible range of one to five among those with no more than two missing items. Higher scores indicate poorer quality of the child's sleep and data were stratified into quartile groups.

\section{Child's hospitalisations}

A hospital admission rate was calculated by dividing the total number of reported hospitalisations by the child's age. Data were then stratified into quartile groups.

\section{Primary caregiver wellbeing}

The SF-12v2 is a valid and reliable instrument of assessing self-reported health and wellbeing [20]. It comprises twelve items that form two scales, the Physical Component Summary (PCS) and Mental Component Summary (MCS), and eight subscales, including Physical Functioning, Role Physical, Bodily Pain, General Health, Vitality, Social Functioning, Role Emotional and Mental Health [20]. The score is calculated based on a normreferencing method using the US general norms collected in 1998 with a mean score of 50 and standard deviation (SD) of 10. Higher scores indicate better health status. Overall, 149 individuals answered all items, and seven and two families had one and two missing items, respectively. For each of eight subscales, a mean value among those without any missing data was applied to best estimate when the subscale score calculation resulted in failure due to the missing items.

\section{Family quality of life}

The BCFQOL Scale assesses family quality of life, specifically, for those raising children with a disability [21, 22]. The instrument, originally developed in the US, has been used in other countries including Australia and Spain [21]. It comprises 25 items with five subscales: Family interaction; Parenting; Emotional wellbeing; Physical/ Material wellbeing; and Disability-related support [22]. The average of total item scores is obtained for each factor, with a possible value of one to five (three and four are rated when a family are neither satisfied or dissatisfied and satisfied with an item, respectively). Higher scores indicate better family quality of life. In total, 130 families responded to every item, and 16 and three individuals had one and two missing items, respectively.

\section{Analysis}

The PCS and MCS scores of the SF-12v2, and five factor scores of the BCFQOL Scale were the dependent variables. Univariate linear regression analyses were performed to investigate their crude relationships with the independent variables including family aspects such as parent's age and employment status, child's characteristics such as current functional ability and SDSC scores, and use of social supports such as respite care services. Multivariate linear regression analyses aimed to examine effects of the child's clinical symptoms and socioenvironmental factors on the SF-12v2 and BCFQOL scores and included adjustment for potential confounders, including socio-demographic factors, family composition and child's age. These variables comprised parent's age, highest qualification and work status, as well as child's age, birth order, number of siblings, and country of birth. Age was a continuous variable and qualification and country of birth were binary variables (i.e. university degree or lower, North America or others, respectively) in the models. Multivariate linear regression models for subscales of the BCFQOL Scale included 
the PCS and MCS as continuous variables as well as all the abovementioned adjustors because the SF-12v2 and BCFQOL scale were scored by the same family member. Differences in mean PCS and MCS scores between 141 biological mothers and the US female norms were assessed using t-tests. The statistical package Stata 14 (StataCorp LP, College Station, TX) was used for all the analyses.

\section{Results}

Table 1 shows the child and family characteristics if the child had a confirmed pathogenic mutation in the CDKL5 gene, regardless of whether they fulfilled inclusion criteria for the analyses. At ascertainment, biological parents ranged in age from 24.6 to 63.7 years with a median of 38.5 years. Approximately $60 \%$ (59.4\%) had university degrees, whereas nearly half (47.5\%) were full-time homemakers or unemployed. The child's median age was 5.2 years, varying from 0.2 to 34.1 years, and the majority $(85.4 \%)$ were females. Over half of the children (52.4\%) were born in the US, followed by the United Kingdom (UK) and Australia (both 7.3\%). More than three quarters $(77.2 \%)$ had siblings and nearly half (46.3\%) were the first child for the parents. Each of the following mutation groups (no functional protein, missense or in-frame mutations within kinase domain, and truncations from aa172 to aa781) accounted for between a quarter and a third of the cases.

Over $60 \%(61.8 \%)$ of those aged 1.5 years or more were able to sit on the floor without any assistance, whereas only $21.3 \%$ were able to walk independently. Less than one fifth (19.6\%) were able to communicate using sign or spoken language and approximately a half (53.8\%) avoided eye contact. Almost two thirds (64.1\%) currently had epileptic seizures on a daily basis. Almost one sixth $(15.8 \%)$ of the children were dependent on enteral nutrition and the majority ( 25 of 27 ) of this group had a gastrostomy. More than half (52.5\%) of mothers of those whose children were orally fed, including combination use with enteral feeding, were concerned about the amount of food and/or liquid their child consumed. Almost three quarters (73.2\%) of the families had utilised a form of respite care services and with nearly a third (32.7\%) receiving both the informal and public services. Nearly half (43.5\%) had experienced financial hardship to meet their child's healthcare needs.

\section{Primary caregiver wellbeing}

The mean PCS score was 53.7 (SD 8.6) ranging from 28.0 to 72.7 . The univariate analysis identified that the child's country of birth had the greatest variability in the scores such that mothers of children who were born in Australia and New Zealand, and Western European countries had lower scores than those of children who were born in North America (coefficients $=-10.98$ and -9.21; $p<0.001$ and $p<0.001$, respectively) (Table 2). Those whose child was dependent on enteral nutrition had considerably poorer physical health (mean PCS score 49.6) compared with those whose child fed orally (mean PCS score 54.3; coefficient $=-4.72 ; p=0.013$ ), as had part-time workers (mean PCS score 50.4) compared with full-time homemakers (mean PCS score 55.1; coefficient $=-4.69 ; p=0.006$ ). The negative effect of enteral feeding remained significant in the multivariate analysis (coefficient $=-4.51 ; p=0.015)$ (Table 3$)$. There was slight improvement in primary caregiver's physical health for those who used both forms of respite care (coefficient = 1.00; $p=0.603)$.

The mean MCS score was 41.9 (SD 11.6) ranging from 9.3 to 63.2. The number of children in the family, followed by the parent's age, had the two greatest impacts in the univariate regression analysis (Table 2). Mothers with an only child having the disorder reported the poorest mental wellbeing with a mean of 37.4, compared with having two, and three or more children (coefficient $=-4.47$ and $-7.56 ; p=0.061$ and 0.002 , respectively). MCS scores rose with increasing parent's age, with the mean score of 41.9 for those aged 30 to 40 years and 43.3 for those aged 40 years or more, compared with that of 35.9 in those aged younger than 30 years $(p=0.101$ and 0.048 , respectively). Child factors also impacted greatly on the MCS scores. Severity of the child's sleep disturbances associated negatively with a mean of 38.2 in the highest quartile (i.e. the greatest difficulty) to 45.2 for the lowest (i.e. the least difficulty) ( $p=0.010)$. Mothers of children who were totally dependent on enteral nutrition had the highest MCS with a mean of 47.4, significantly higher than those whose children were totally orally fed $(p=0.013)$. Experiencing financial hardship adversely affected mental health (coefficient $=-4.89 ; p=0.011$ ). The multivariate analysis identified that the child's sleep disturbances had the largest negative effect on MCS scores (coefficient $=-8.04 ; p=0.008$, the highest vs. lowest quartile) (Table 3). The impact of the child's feeding pattern and financial difficulty experiences remained considerable. Utilisation of respite care services did not appear to be associated with improvement in primary caregiver's emotional health.

The mean PCS score of 141 of biological mothers was higher overall at 54.0 (SD 8.5), compared with 48.5 (SD 9.9) for the US female norms $(p<0.001)$, but for those aged 25 to 34 years the increase was minimal at 1.4 points $(95 \% \mathrm{CI}-1.29,4.13)$. On the other hand, the mean MCS score for CDKL5 mothers at 41.5 (SD 11.5), was 6.90 points $(95 \% \mathrm{CI}-8.57,-5.23)$ lower that of the US female norms $(p<0.001)$. The difference was virtually consistent across the age groups. 
Table 1 Family demographics and the child's characteristics in the database $(n=192)$

\begin{tabular}{|c|c|c|c|}
\hline \multicolumn{2}{|l|}{ Family factors } & \multicolumn{2}{|l|}{$\overline{\text { Floor sitting }(n=144)^{\# 5}, n(\%)}$} \\
\hline Parental age $(n=160)$ mean (SD), years & $38.2(7.0)$ & No assistance & $89(61.8)$ \\
\hline$n(\%)$ & & Some assistance & $14(9.7)$ \\
\hline Under 30 years & $14(8.8)$ & Mximal assistance/unable & $41(28.5)$ \\
\hline 30 to 40 years & $84(52.5)$ & \multicolumn{2}{|l|}{ Taking 10 steps forward $(n=141)^{\# 5}, n(\%)$} \\
\hline 40 years or over & $62(38.8)$ & No assistance & $30(21.3)$ \\
\hline \multicolumn{2}{|l|}{ Parental qualification ( $n=160), n(\%)$} & Some assistance & $20(14.2)$ \\
\hline 11 or 12 years of school & $34(21.3)$ & Mximal assistance/unable & $91(64.5)$ \\
\hline TAFE/technical certificate & $31(19.4)$ & \multicolumn{2}{|l|}{ Eye contact $(n=158), n(\%)$} \\
\hline University degree & $95(59.4)$ & No & $85(53.8)$ \\
\hline \multicolumn{2}{|l|}{ Parental work status ( $n=160), n(\%)$} & Yes & $73(46.2)$ \\
\hline Full-time homemaker/unemployment & $76(47.5)$ & \multicolumn{2}{|l|}{ Communication $(n=143)^{\# 5}, n(\%)$} \\
\hline Part-time employment & $39(24.4)$ & No/Simple communication & $53(37.1)$ \\
\hline Full-time employment & $45(28.1)$ & Complex gestures/vocalisation & $62(43.4)$ \\
\hline \multicolumn{2}{|l|}{ Number of siblings ( $n=162), n(\%)$} & Sign/Spoken language & $28(19.6)$ \\
\hline 0 & $37(22.8)$ & \multicolumn{2}{|l|}{ Seizure frequency $(n=160), n(\%)$} \\
\hline 1 & $67(41.4)$ & Absent & $14(8.8)$ \\
\hline 2 or more & $58(35.8)$ & Yearly/Monthly & $16(10.0)$ \\
\hline \multicolumn{2}{|l|}{ Birth order $(n=162), n(\%)$} & Weekly & $27(16.9)$ \\
\hline The first child & $75(46.3)$ & 1 to 5 times per day & $74(46.3)$ \\
\hline Laterborn & $87(53.7)$ & At least 5 times per day & $29(18.1)$ \\
\hline Child factors & & \multicolumn{2}{|c|}{ Sleep difficulties (average SDSC score) ( $n=155), n(\%)$} \\
\hline Child's age $(n=192)$ mean $(S D)$, years & $7.1(6.1)$ & 1 to 1.50 ( $1^{\text {st }}$ quartile $)$ & $38(24.5)$ \\
\hline \multicolumn{2}{|l|}{$n(\%)$} & 1.50 to 1.81 ( $2^{\text {nd }}$ quartile) & $39(25.2)$ \\
\hline Under 1.5 years & $30(15.6)$ & 1.81 to 2.17 ( $3^{\text {rd }}$ quartile) & $40(25.8)$ \\
\hline 1.5 to 7 years & $86(44.8)$ & 2.17 to 5 ( $4^{\text {th }}$ quartile) & $38(24.5)$ \\
\hline 7 to 13 years & $48(25.0)$ & \multicolumn{2}{|c|}{ Hospitalisation rate $(n=156)$, per 1 person-year, $n(\%)$} \\
\hline 13 years & $28(14.6)$ & 0 to 0.38 ( $1^{\text {st }}$ quartile $)$ & $39(25.0)$ \\
\hline \multicolumn{2}{|l|}{ Child's gender ( $n=192), n(\%)$} & 0.38 to 0.86 ( $2^{\text {nd }}$ quartile) & $39(25.0)$ \\
\hline Female & $164(85.4)$ & 0.86 to 2.14 ( $3^{\text {rd }}$ quartile) & $39(25.0)$ \\
\hline Male & $28(14.6)$ & 2.14 to 13.70 (4th quartile) & $39(25.0)$ \\
\hline \multicolumn{2}{|l|}{ Child's country of birth $(n=191), n(\%)$} & \multicolumn{2}{|l|}{ Socio-environmental factors } \\
\hline North America & $105(55.0)$ & \multicolumn{2}{|l|}{ Respite care $(n=153), n(\%)$} \\
\hline The United Kingdom & $14(7.3)$ & None & $41(26.8)$ \\
\hline Australia and New Zealand & $16(8.4)$ & Formal respite care only & $21(13.7)$ \\
\hline Western Europe & $38(19.9)$ & Informal respite care only & $41(26.8)$ \\
\hline Others & $18(9.4)$ & & \\
\hline \multicolumn{2}{|l|}{ Mutation group ( $n=192), n(\%)$} & Both formal and informal respite care & $50(32.7)$ \\
\hline No functional protein & $55(28.7)$ & \multicolumn{2}{|l|}{ Financial difficulty ( $n=147), n(\%)$} \\
\hline Missense/In-frame mutations within kinase domain & $48(25.0)$ & No & $83(56.5)$ \\
\hline Truncations from aa172 to aa781 & $58(30.2)$ & Yes & $64(43.5)$ \\
\hline Truncations after aa781 & $16(8.3)$ & \multirow{10}{*}{\multicolumn{2}{|c|}{$\begin{array}{l}\# 1 \text { North America includes the United States (100) and Canada (5). Australia } \\
\text { and New Zealand contains Australia (14) and New Zealand (2). Western Europe } \\
\text { includes Germany (12), France (7), the Netherlands (6), Ireland (3), Spain (2), } \\
\text { Italy (2), Belgium (1), Denmark (1), Finland (1), Norway (1), Sweden (1) and } \\
\text { Luxemburg (1). Others comprises Brazil (3), India (2), Russia (2), Hungary (2), } \\
\text { Bulgaria (1), Poland (1), Slovenia (1), Israel (1), China (1), Singapore (1), Mexico } \\
\text { (1), Perto Rico (1) and Argentina (1). } \\
\# 214 \text { used a gastrostomy tube, } 2 \text { used a nasogastric tube } \\
\# 325 \text { used a gastrostomy tube, } 2 \text { used a nasogastric tube } \\
\# 4 \text { Among children who intook food orally with or without combination use of } \\
\text { external nutrition } \\
\# 5 \text { Excludes child aged younger than } 1.5 \text { years }\end{array}$}} \\
\hline Mutations not grouped & $15(7.8)$ & & \\
\hline \multicolumn{2}{|l|}{ Feeding pattern $(n=171), n(\%)$} & & \\
\hline Oral intake only & $128(74.9)$ & & \\
\hline Combination use $\mathrm{e}^{\# 2}$ & $16(9.4)$ & & \\
\hline Enteral nutrition only ${ }^{\# 3}$ & $27(15.8)$ & & \\
\hline \multicolumn{2}{|l|}{ Dietary concerns $(n=139)^{\# 4}, n(\%)$} & & \\
\hline None & $66(47.5)$ & & \\
\hline Occasional & $47(33.8)$ & & \\
\hline Frequent/constant & $26(18.7)$ & & \\
\hline
\end{tabular}

Table 1 Family demographics and the child's characteristics in the database ( $n=192)$ (Continued)

$$
\text { the database }(n=192) \text { (Continued) }
$$

$4(9.7)$

Mximal assistance/unable 
Table 2 Univariate analysis for PCS and MCS of the SF-12V2 $(n=158)$

\begin{tabular}{|c|c|c|c|c|c|}
\hline \multirow[t]{2}{*}{ Measure } & \multirow{2}{*}{$\begin{array}{l}\text { Number of } \\
\text { observations }\end{array}$} & \multicolumn{2}{|l|}{ PCS } & \multicolumn{2}{|l|}{ MCS } \\
\hline & & Mean (SD) 53.7 (8.6) & $\overline{\text { Co-efficient }}$ & Mean (SD) 41.9 (11.6) & Co-efficient \\
\hline Parent's age & 152 & & & & \\
\hline Under 30 years & 12 & $53.5(5.9)$ & Reference & $35.9(15.7)$ & Reference \\
\hline 30 to 40 years & 80 & $54.7(8.7)$ & 1.23 & $41.9(11.3)$ & 5.97 \\
\hline 40 years or over & 60 & $52.9(8.7)$ & -0.59 & $43.3(11.3)$ & $7.37^{* *}$ \\
\hline Parent's qualification & 147 & & & & \\
\hline 11 or 12 years of school & 27 & $53.2(7.9)$ & Reference & $41.8(13.2)$ & Reference \\
\hline TAFE/Technical certificate & 29 & $53.6(9.3)$ & 0.40 & $41.2(9.0)$ & -0.57 \\
\hline University degree & 91 & $54.4(8.4)$ & 1.14 & $42.0(12.1)$ & -0.23 \\
\hline Maternal work status & 147 & & & & \\
\hline Full-time homemaker & 64 & $55.1(8.1)$ & Reference & $42.7(11.3)$ & Reference \\
\hline Part-time employment & 37 & $50.4(9.4)$ & $-4.69^{* *}$ & $42.4(11.9)$ & -0.32 \\
\hline Full-time employment & 46 & $55.7(7.5)$ & 0.57 & $40.8(11.2)$ & -1.86 \\
\hline Number of siblings & 156 & & & & \\
\hline 0 & 36 & $54.3(8.1)$ & Reference & $37.4(13.8)$ & Reference \\
\hline 1 & 64 & $54.4(7.5)$ & 0.07 & $41.9(11.3)$ & $4.47^{*}$ \\
\hline 2 or more & 56 & $52.9(9.9)$ & -1.41 & $45.0(9.6)$ & $7.56^{* *}$ \\
\hline Child order & 156 & & & & \\
\hline The first child & 72 & $54.4(7.9)$ & Reference & $40.2(11.7)$ & Reference \\
\hline Laterborn & 84 & $53.3(9.1)$ & -1.02 & $43.5(11.4)$ & $3.24^{*}$ \\
\hline Child's age & 158 & & & & \\
\hline Under 1.5 years & 24 & $53.3(10.0)$ & Reference & $39.8(13.2)$ & Reference \\
\hline 1.5 to 7 years & 69 & $54.6(7.6)$ & 1.31 & $42.4(12.4)$ & 2.63 \\
\hline 7 to 13 years & 40 & $52.7(9.8)$ & -0.63 & $43.7(9.7)$ & 3.94 \\
\hline 13 years or over & 25 & $53.0(8.0)$ & -0.29 & $39.2(10.7)$ & -0.55 \\
\hline Child's gender & 158 & & & & \\
\hline Female & 136 & $53.9(8.3)$ & Reference & $41.8(11.6)$ & Reference \\
\hline Male & 22 & $52.3(10.4)$ & -1.60 & $41.9(12.1)$ & 0.10 \\
\hline Child's country of birth & 158 & & & & \\
\hline North America & 90 & $56.9(5.9)$ & Reference & $41.5(12.1)$ & Reference \\
\hline The United Kingdom & 12 & $54.2(10.9)$ & -2.63 & $41.1(8.4)$ & -0.40 \\
\hline Australia and New Zealand & 14 & $45.9(7.4)$ & $-10.98^{* *}$ & $43.6(12.5)$ & 2.07 \\
\hline Western Europe & 29 & $47.6(10.0)$ & $-9.21^{* *}$ & $41.2(12.2)$ & -0.30 \\
\hline Others & 13 & $52.9(8.2)$ & $-3.93^{*}$ & $44.5(9.7)$ & 3.02 \\
\hline Mutation group & 158 & & & & \\
\hline No functional protein & 50 & $53.5(8.0)$ & Reference & $43.0(12.0)$ & Reference \\
\hline Missense/In-frame mutations & 41 & $52.5(9.9)$ & -0.97 & $39.5(10.8)$ & -3.49 \\
\hline Truncations aa172 to aa781 & 44 & $53.7(8.7)$ & 0.23 & $42.0(11.5)$ & -0.98 \\
\hline Truncations after aa781 & 12 & $53.6(7.4)$ & 0.15 & $46.2(9.1)$ & 3.21 \\
\hline Mutations not grouped & 11 & $58.5(6.5)$ & $5.05^{*}$ & $39.5(15.1)$ & -3.49 \\
\hline Feeding pattern & 153 & & & & \\
\hline Oral intake only & 114 & $54.3(8.1)$ & Reference & $41.0(12.2)$ & Reference \\
\hline Combination use & 14 & $55.0(6.8)$ & 0.74 & $39.7(9.4)$ & -1.32 \\
\hline Enteral nutrition only & 25 & $49.6(11.0)$ & $-4.72^{* *}$ & $47.4(9.3)$ & $6.37^{* *}$ \\
\hline
\end{tabular}


Table 2 Univariate analysis for PCS and MCS of the SF-12v2 $(n=158)$ (Continued)

\begin{tabular}{|c|c|c|c|c|c|}
\hline Dietary concerns & 123 & & & & \\
\hline None & 62 & $55.9(7.3)$ & Reference & $41.9(11.7)$ & Reference \\
\hline Occasional & 39 & $51.8(7.4)$ & $-4.14^{* *}$ & $39.9(12.6)$ & -2.00 \\
\hline Frequent or constant & 22 & $55.7(8.0)$ & -0.25 & $42.5(10.3)$ & 0.67 \\
\hline Floor sitting & 129 & & & & \\
\hline No assistance & 83 & $54.3(7.6)$ & Reference & $41.7(11.7)$ & Reference \\
\hline Some assistance & 13 & $54.5(7.8)$ & 0.20 & $42.0(12.5)$ & 0.27 \\
\hline Maximal assistance/unable & 33 & $51.3(10.3)$ & $-3.02^{*}$ & $43.9(10.6)$ & 2.25 \\
\hline Taking 10 steps forward & 126 & & & & \\
\hline No assistance & 28 & $54.8(7.2)$ & Reference & $41.6(12.6)$ & Reference \\
\hline Some assistance & 19 & $57.3(7.2)$ & 2.52 & $40.6(14.3)$ & -0.95 \\
\hline Maximal assistance/unable & 79 & $52.4(8.9)$ & -2.37 & $43.2(10.4)$ & 1.63 \\
\hline Eye contact & 148 & & & & \\
\hline No & 78 & $53.0(9.6)$ & Reference & $41.0(12.0)$ & Reference \\
\hline Yes & 70 & $54.6(7.3)$ & 1.57 & $42.5(11.7)$ & 1.54 \\
\hline Communication & 129 & & & & \\
\hline No/Simple method & 44 & $53.0(9.0)$ & Reference & $44.1(9.6)$ & Reference \\
\hline Complex gestures/vocalisation & 58 & $53.5(8.5)$ & 0.52 & $41.2(12.4)$ & -2.91 \\
\hline Sign/Spoken language & 27 & $55.0(7.5)$ & 1.98 & $42.1(12.0)$ & -2.02 \\
\hline Seizure frequency & 146 & & & & \\
\hline Absent & 12 & $53.7(7.5)$ & Reference & $43.2(11.3)$ & Reference \\
\hline Yearly/Monthly & 15 & $53.4(9.8)$ & -0.33 & $41.3(13.1)$ & -1.82 \\
\hline Weekly & 26 & $54.5(6.7)$ & 0.84 & $40.5(10.7)$ & -2.71 \\
\hline 1 to 5 times a day & 69 & $53.4(8.9)$ & -0.28 & $41.1(12.7)$ & -2.09 \\
\hline At least 5 times a day & 24 & $54.2(9.8)$ & 0.49 & $45.6(8.5)$ & 2.47 \\
\hline Sleep difficulties & 144 & & & & \\
\hline 1.00 to 1.50 ( $1^{\text {st }}$ quartile) & 36 & $54.5(8.3)$ & Reference & $45.2(11.3)$ & Reference \\
\hline 1.50 to 1.81 ( $2^{\text {nd }}$ quartile) & 38 & $54.1(7.7)$ & -0.46 & $42.7(10.8)$ & -2.58 \\
\hline 1.81 to 2.17 ( $3^{\text {rd }}$ quartile) & 35 & $54.6(7.5)$ & 0.11 & $42.6(11.2)$ & -2.63 \\
\hline 2.17 to 5.00 ( $4^{\text {th }}$ quartile) & 35 & $52.5(10.1)$ & -2.00 & $38.2(12.1)$ & $-7.06^{* *}$ \\
\hline Hospitalisation rate & 146 & & & & \\
\hline 0 to 0.38 ( $1^{\text {st }}$ quartile) & 34 & $52.9(7.8)$ & Reference & $42.5(10.2)$ & Reference \\
\hline 0.38 to 0.86 ( $2^{\text {nd }}$ quartile $)$ & 38 & $54.6(8.3)$ & 1.66 & $43.0(12.2)$ & 0.50 \\
\hline 0.86 to 2.14 ( $3^{\text {rd }}$ quartile) & 36 & $53.1(8.4)$ & 0.23 & $39.9(10.3)$ & -2.60 \\
\hline 2.14 to 13.70 ( $4^{\text {th }}$ quartile) & 38 & $54.0(9.7)$ & 1.11 & $43.7(12.2)$ & 1.19 \\
\hline Respite care & 148 & & & & \\
\hline None & 36 & $54.4(8.3)$ & Reference & $42.7(11.8)$ & Reference \\
\hline Formal only & 21 & $52.0(10.1)$ & -2.41 & $44.9(8.3)$ & 2.21 \\
\hline Informal only & 41 & $54.1(8.6)$ & -0.26 & $41.1(13.9)$ & -1.56 \\
\hline Both formal and informal & 50 & $53.2(8.4)$ & -1.25 & $40.8(10.4)$ & -1.85 \\
\hline Financial difficulty & 144 & & & & \\
\hline No & 83 & $53.9(8.5)$ & Reference & $44.0(11.2)$ & Reference \\
\hline Yes & 61 & $52.9(8.9)$ & -0.97 & $39.1(11.6)$ & $-4.89^{* *}$ \\
\hline
\end{tabular}

${ }^{*} p<0.10{ }^{* *} p<0.05$ 
Table 3 Multivariate analysis for PCS and MCS of the SF-12v2 and coefficients ${ }^{\# 1}$

\begin{tabular}{|c|c|c|c|}
\hline \multirow[t]{2}{*}{ Measure } & & \multirow{2}{*}{$\begin{array}{l}\text { PCS } \\
\text { Coefficient }(95 \% \mathrm{Cl})\end{array}$} & \multirow{2}{*}{$\begin{array}{l}\text { MCS } \\
\text { Coefficient }(95 \% \text { Cl) }\end{array}$} \\
\hline & & & \\
\hline \multirow[t]{3}{*}{ Feeding pattern } & Oral intake only & Reference & Reference \\
\hline & Combination use & $-1.01(-5.63,3.60)$ & $-1.49(-8.36,5.37)$ \\
\hline & Enteral nutrition only & $-4.51^{* *}(-8.12,-0.89)$ & $6.67^{* *}$ (1.29 to 12.05$)$ \\
\hline \multirow[t]{3}{*}{ Dietary concerns } & None & Reference & Reference \\
\hline & Occasional & $-4.98^{* *}(-8.11,-1.86)$ & $-0.88(-5.97,4.20)$ \\
\hline & Frequent or constant & $-1.81(-5.51,1.88)$ & $0.30(-5.72,6.32)$ \\
\hline \multirow[t]{3}{*}{ Floor sitting } & No assistance & Reference & Reference \\
\hline & Some assistance & $0.21(-4.01,4.42)$ & $-1.59(-8.14,4.96)$ \\
\hline & Maximal assistance/unable & $-2.27(-5.44,0.90)$ & $1.03(-3.89,5.96)$ \\
\hline \multirow[t]{3}{*}{ Taking 10 steps forward } & No assistance & Reference & Reference \\
\hline & Some assistance & $1.20(-3.52,5.91)$ & $-1.23(-8.59,6.14)$ \\
\hline & Maximal assistance/unable & $-1.85(-5.34,1.64)$ & $0.15(-5.30,5.61)$ \\
\hline \multirow[t]{2}{*}{ Eye contact } & No & Reference & Reference \\
\hline & Yes & $1.04(-1.67,3.75)$ & $1.77(-2.46,6.00)$ \\
\hline \multirow[t]{3}{*}{ Communication } & No/Simple method & Reference & Reference \\
\hline & Complex gestures/vocalisation & $0.45(-2.58,3.48)$ & $-0.85(-5.42,3.72)$ \\
\hline & Sign/Spoken language & $1.03(-3.00,5.06)$ & $0.14(-5.93,6.21)$ \\
\hline \multirow[t]{5}{*}{ Seizure frequency } & Absent & Reference & Reference \\
\hline & Yearly/Monthly & $-0.55(-6.78,5.68)$ & $-2.82(-12.11,6.46)$ \\
\hline & Weekly & $0.42(-5.41,6.25)$ & $-3.42(-12.11,5.27)$ \\
\hline & 1 to 5 times a day & $-0.76(-5.88,4.35)$ & $-1.90(-9.52,5.73)$ \\
\hline & At least 5 times a day & $-0.17(-6.07,5.72)$ & $0.09(-8.69,8.88)$ \\
\hline \multirow[t]{4}{*}{ Sleep difficulties } & 1.00 to 1.50 ( $1^{\text {st }}$ quartile) & Reference & Reference \\
\hline & 1.50 to 1.81 ( $2^{\text {nd }}$ quartile) & $0.37(-3.31,4.04)$ & $-2.58(-8.11,2.95)$ \\
\hline & 1.81 to 2.17 ( $3^{\text {rd }}$ quartile) & $2.12(-1.65,5.89)$ & $-2.57(-8.25,3.10)$ \\
\hline & 2.17 to 5.00 ( $4^{\text {th }}$ quartile) & $1.02(-2.88,4.93)$ & $-8.04^{* *}(-13.91,-2.16)$ \\
\hline \multirow[t]{4}{*}{ Hospitalisation rate } & 0 to 0.38 ( $1^{\text {st }}$ quartile) & Reference & Reference \\
\hline & 0.38 to 0.86 ( $2^{\text {nd }}$ quartile $)$ & $-0.60(-4.49,3.30)$ & $-1.13(-7.01,4.73)$ \\
\hline & 0.86 to 2.14 ( $3^{\text {rd }}$ quartile) & $-2.27(-6.42,1.87)$ & $-4.22(-10.47,2.03)$ \\
\hline & 2.14 to 13.70 (4h quartile) & $-1.15(-5.31,3.00)$ & $-0.88(-7.15,5.38)$ \\
\hline \multirow[t]{4}{*}{ Respite care } & None & Reference & Reference \\
\hline & Formal only & $0.08(-4.63,4.79)$ & $1.54(-5.63,8.69)$ \\
\hline & Informal only & $-0.80(-4.57,2.97)$ & $-1.54(-7.26,4.18)$ \\
\hline & Both formal and informal & $1.00(-2.79,4.78)$ & $-2.73(-8.48,3.02)$ \\
\hline \multirow[t]{2}{*}{ Financial difficulty } & No & Reference & Reference \\
\hline & Yes & $-1.76(-4.43,0.92)$ & $-4.75^{* *}(-8.78,-0.71)$ \\
\hline
\end{tabular}

\#1Adjusted for parent's age (a continuous variable), parent's qualification (a binary variable; university degree or lower), parent's work status, number of siblings, the first child, child's age (a continuous variable) and child's country of birth (a binary variable; North America or others)

** $p<0.05$

\section{Family quality of life}

Family quality of life was generally rated as satisfactory with an overall score of 4.06 (SD 0.66). Among the subscales, emotional wellbeing scores were lowest with a mean of 3.50 (SD 0.97) and physical/material wellbeing score were the highest with a mean of 4.32 (SD 0.70) (Table 4). The univariate analysis demonstrated that the child's country of birth profoundly influenced the BCFQOL scores, in which those from Western Europe except the UK had the poorest family quality of life 
Table 4 Univariate analysis for the BCFQOL Scale and coefficients $(n=149)$

\begin{tabular}{|c|c|c|c|c|c|c|}
\hline \multirow[t]{2}{*}{ Measure } & \multirow[t]{2}{*}{$\begin{array}{l}\text { Number of } \\
\text { observations }\end{array}$} & \multirow{2}{*}{$\begin{array}{l}\text { Family interaction } \\
\text { Mean (SD) } \\
4.22(0.71)\end{array}$} & \multirow{2}{*}{$\begin{array}{l}\text { Parenting } \\
\text { Mean (SD) } \\
4.15(0.72)\end{array}$} & \multirow{2}{*}{$\begin{array}{l}\text { Emotional wellbeing } \\
\text { Mean (SD) } \\
3.50(0.97)\end{array}$} & \multirow{2}{*}{$\begin{array}{l}\text { Physical/Material } \\
\text { wellbeing } \\
\text { Mean (SD) } \\
4.32(0.70)\end{array}$} & \multirow{2}{*}{$\begin{array}{l}\text { Disability-related } \\
\text { support } \\
\text { Mean (SD) } \\
4.13(0.77)\end{array}$} \\
\hline & & & & & & \\
\hline Parent's age & 143 & & & & & \\
\hline Under 30 years & 11 & Reference & Reference & Reference & Reference & Reference \\
\hline 30 to 40 years & 75 & -0.18 & -0.13 & 0.07 & 0.07 & $-0.51^{* *}$ \\
\hline 40 years or over & 57 & $-0.45^{*}$ & -0.34 & 0.06 & -0.06 & $-0.71^{* *}$ \\
\hline Parent's qualification & 137 & & & & & \\
\hline 11 or 12 years of school & 24 & Reference & Reference & Reference & Reference & Reference \\
\hline TAFE/Technical certificate & 28 & 0.18 & 0.10 & 0.05 & $0.36^{*}$ & -0.11 \\
\hline University degree & 85 & 0.13 & 0.07 & 0.07 & $0.28^{*}$ & -0.11 \\
\hline Parent's work status & 138 & & & & & \\
\hline Full-time homemaker & 58 & Reference & Reference & Reference & Reference & Reference \\
\hline Part-time employment & 34 & -0.21 & $-0.32^{* *}$ & 0.18 & -0.19 & -0.24 \\
\hline Full-time employment & 46 & 0.03 & -0.06 & 0.29 & -0.03 & -0.24 \\
\hline Number of siblings & 147 & & & & & \\
\hline 0 & 29 & Reference & Reference & Reference & Reference & Reference \\
\hline 1 & 64 & -0.10 & 0.09 & -0.17 & 0.13 & -0.03 \\
\hline 2 or more & 54 & 0.04 & 0.20 & -0.10 & 0.25 & 0.11 \\
\hline Child order & 147 & & & & & \\
\hline The first child & 63 & Reference & Reference & Reference & Reference & Reference \\
\hline Laterborn & 84 & 0.00 & 0.10 & 0.06 & 0.17 & 0.05 \\
\hline Child's age & 149 & & & & & \\
\hline Under 1.5 years & 21 & Reference & Reference & Reference & Reference & Reference \\
\hline 1.5 to 7 years & 67 & 0.06 & -0.11 & -0.32 & -0.11 & 0.09 \\
\hline 7 to 13 years & 38 & 0.00 & -0.12 & -0.35 & -0.11 & 0.05 \\
\hline 13 years or over & 23 & -0.21 & -0.34 & -0.28 & $-0.36^{*}$ & -0.09 \\
\hline Child's gender & 149 & & & & & \\
\hline Female & 128 & Reference & Reference & Reference & Reference & Reference \\
\hline Male & 21 & 0.13 & 0.07 & 0.00 & 0.01 & -0.12 \\
\hline Child's country of birth & 149 & & & & & \\
\hline North America & 87 & Reference & Reference & Reference & Reference & Reference \\
\hline The United Kingdom & 12 & -0.02 & -0.09 & -0.05 & -0.20 & -0.25 \\
\hline Australia and New Zealand & 13 & -0.29 & $-0.48^{* *}$ & -0.21 & $-0.45^{* *}$ & -0.19 \\
\hline Western Europe & 26 & $-0.51^{* *}$ & $-0.52^{* *}$ & $-0.43^{*}$ & $-0.46^{* *}$ & $-0.61^{* *}$ \\
\hline Others & 11 & -0.01 & -0.18 & -0.30 & -0.32 & -0.22 \\
\hline Mutation group & 149 & & & & & \\
\hline No functional protein & 46 & Reference & Reference & Reference & Reference & Reference \\
\hline Missense/In-frame mutations & 36 & 0.10 & 0.03 & -0.16 & 0.01 & -0.16 \\
\hline Truncations aa172 to aa781 & 45 & 0.05 & -0.06 & -0.16 & 0.02 & -0.08 \\
\hline Truncations after aa781 & 12 & 0.07 & 0.06 & 0.11 & 0.16 & 0.06 \\
\hline Mutations not grouped & 10 & -0.01 & -0.17 & -0.06 & -0.13 & -0.31 \\
\hline Feeding pattern & 144 & & & & & \\
\hline Oral intake only & 107 & Reference & Reference & Reference & Reference & Reference \\
\hline Combination use & 14 & -0.13 & -0.16 & -0.04 & $-0.34^{*}$ & -0.10 \\
\hline Enteral nutrition only & 23 & 0.21 & 0.15 & 0.15 & -0.02 & 0.06 \\
\hline
\end{tabular}


Table 4 Univariate analysis for the BCFQOL Scale and coefficients $(n=149)$ (Continued)

\begin{tabular}{|c|c|c|c|c|c|c|}
\hline Dietary concerns & 117 & & & & & \\
\hline None & 57 & Reference & Reference & Reference & Reference & Reference \\
\hline Occasional & 38 & -0.22 & $-0.34^{* *}$ & $-0.40^{*}$ & $-0.40^{* *}$ & $-0.32^{* *}$ \\
\hline Frequent or constant & 22 & 0.18 & -0.20 & -0.02 & -0.14 & -0.20 \\
\hline Floor sitting & 123 & & & & & \\
\hline No assistance & 77 & Reference & Reference & Reference & Reference & Reference \\
\hline Some assistance & 13 & 0.07 & 0.07 & 0.29 & -0.04 & 0.22 \\
\hline Maximal assistance/unable & 33 & 0.17 & 0.19 & 0.15 & 0.06 & 0.15 \\
\hline Taking 10 steps forward & 20 & & & & & \\
\hline No assistance & 26 & Reference & Reference & Reference & Reference & Reference \\
\hline Some assistance & 17 & 0.15 & -0.05 & -0.03 & -0.07 & -0.04 \\
\hline Maximal assistance/unable & 77 & 0.16 & 0.01 & 0.06 & -0.02 & 0.01 \\
\hline Eye contact & 139 & & & & & \\
\hline No & 71 & Reference & Reference & Reference & Reference & Reference \\
\hline Yes & 68 & -0.10 & -0.11 & -0.19 & -0.09 & -0.05 \\
\hline Communication & 123 & & & & & \\
\hline No/Simple method & 42 & Reference & Reference & Reference & Reference & Reference \\
\hline Complex gestures/vocalisation & 57 & -0.16 & -0.18 & $-0.38^{*}$ & -0.16 & -0.24 \\
\hline Sign/Spoken language & 24 & 0.06 & 0.10 & -0.02 & 0.19 & 0.18 \\
\hline Seizure frequency & 138 & & & & & \\
\hline Absent & 12 & Reference & Reference & Reference & Reference & Reference \\
\hline Yearly/Monthly & 15 & -0.28 & -0.15 & -0.07 & -0.25 & 0.12 \\
\hline Weekly & 25 & -0.29 & -0.03 & 0.14 & -0.19 & -0.03 \\
\hline 1 to 5 times a day & 62 & -0.15 & -0.00 & 0.11 & -0.09 & 0.09 \\
\hline At least 5 times a day & 24 & -0.11 & 0.14 & 0.39 & -0.06 & 0.22 \\
\hline Sleep difficulties & 136 & & & & & \\
\hline 1.00 to 1.50 ( $1^{\text {st }}$ quartile) & 34 & Reference & Reference & Reference & Reference & Reference \\
\hline 1.50 to 1.81 ( $2^{\text {nd }}$ quartile $)$ & 37 & -0.24 & -0.23 & $-0.42^{*}$ & $-0.33^{* *}$ & $-0.37^{* *}$ \\
\hline 1.81 to 2.17 ( $3^{\text {rd }}$ quartile) & 32 & -0.19 & -0.20 & -0.34 & -0.07 & -0.14 \\
\hline 2.17 to 5.00 ( $4^{\text {th }}$ quartile $)$ & 33 & $-0.34^{*}$ & $-0.34^{*}$ & $-0.57^{* *}$ & $-0.41^{* *}$ & $-0.39^{* *}$ \\
\hline Hospitalisation rate & 139 & & & & & \\
\hline 0 to 0.38 ( $1^{\text {st }}$ quartile) & 31 & Reference & Reference & Reference & Reference & Reference \\
\hline 0.38 to 0.86 ( $2^{\text {nd }}$ quartile) & 36 & 0.19 & 0.17 & 0.13 & 0.15 & 0.25 \\
\hline 0.86 to 2.14 ( $3^{\text {rd }}$ quartile) & 35 & 0.01 & -0.09 & -0.42 & -0.07 & 0.15 \\
\hline 2.14 to 13.70 ( $4^{\text {th }}$ quartile $)$ & 37 & 0.20 & $0.35^{* *}$ & 0.06 & 0.07 & 0.16 \\
\hline Respite care & 140 & & & & & \\
\hline None & 37 & Reference & Reference & Reference & Reference & Reference \\
\hline Formal only & 21 & -0.27 & -0.18 & $-0.56^{* *}$ & -0.16 & -0.15 \\
\hline Informal only & 35 & -0.12 & -0.19 & -0.37 & $-0.27^{*}$ & $-0.31^{*}$ \\
\hline Both formal and informal & 47 & $-0.50^{* *}$ & $-0.35^{* *}$ & -0.24 & $-0.34^{* *}$ & $-0.44^{* *}$ \\
\hline Financial difficulty & 137 & & & & & \\
\hline No & 76 & Reference & Reference & Reference & Reference & Reference \\
\hline Yes & 61 & 0.03 & -0.09 & $-0.57^{* *}$ & $-0.22^{*}$ & -0.09 \\
\hline
\end{tabular}

across subscales. Use of respite services was associated adversely with every subscale, with lower BCFQOL scores in family interaction, parenting, emotional wellbeing, physical/material wellbeing and disability-related support observed for those who had received both forms of the services compared to non-users (coefficient $=-0.50$, 
$-0.35,-0.24,-0.34$ and $-0.44 ; p=0.001,0.028,0.268$, 0.027 and 0.011 , respectively). There was a negative correlation with the SDSC scores such that the families of the children with the greatest sleep difficulties had the lowest scores with all BCFQOL subscales, with the coefficients of -0.34 for family interaction, -0.34 for parenting, -0.57 for emotional wellbeing, -0.41 for physical/material wellbeing and -0.39 for disability-related support compared to the least sleep disturbances $(p=0.054$, $0.057,0.020,0.015$ and 0.040 , respectively). Mothers aged 40 years or more reported considerably poorer quality of disability-related support, family interaction and parenting, compared with those aged under 30 years (coefficients $=-0.71,-0.45$ and -0.34 ; $p=0.005,0.056$ and 0.159 , respectively). The multivariate model identified that those with the most extensive utilisation of respite care had the poorest family quality of life with emotional wellbeing subscale scores being the lowest for those who used formal respite care in comparison with those who had never received such services (Table 5). Particularly, the effects on family interaction, emotional wellbeing, physical/material wellbeing and disability-related support were considerable (coefficients $=-0.39,-0.36,-0.32$ and $-0.39 ; p=0.027,0.127,0.048$ and 0.044 , respectively). The impact of child's sleep difficulty was attenuated after adjusting for PCS and MCS scores.

\section{Discussion}

Caregivers of children with the CDKL5 disorder experienced considerable emotional burden. Despite better physical wellbeing, emotional wellbeing (MCS 41.9, SD 11.6) was compromised in comparison with the US female norms and was poorer than has been demonstrated in Australian research in both Rett syndrome [12] (MCS 42.4, SD 10.2) and Down syndrome [11] (MCS 45.2, SD 10.6). In contrast, a large proportion of mothers in the international CDKL5 disorder sample were from the US, and generally younger than those in the Australian studies $[11,12]$, factors which may also have some bearing on the findings.

Emotional wellbeing of primary care givers was particularly poor when the child was an only child, the child's co-occurring sleep disturbances were severe, and the family had faced financial hardships to meet the child's healthcare needs. These associations are broadly consistent with findings of most previous research. Poor maternal sleep quality, often a sequela of child's sleep disturbances, has been shown to be an important predictor of depression in mothers of children with developmental disabilities, mostly autism spectrum disorder [23-27] or cerebral palsy [28]. Moreover, a similar relationship to that we identified with financial hardship was demonstrated in a Canadian study involving mothers with a child with a developmental disability [29].

Our study reveals some unique findings. Firstly, mental health was least impaired in mothers caring for a child who was totally tube fed, whereas this was the group with the poorest physical health. The literature has shown variability in parent's experiences for those with a child with a disability after the child's gastrostomy placement, although enhancement in nutritional status has been reported [30-33]. Parents have reported less burden of care at mealtimes, reduced stress that their child is inadequately nourished, and relief that medications are consistently delivered, consequently improving their emotional wellbeing [30-34]. In a previous study with primary caregivers of a child with Rett syndrome we found that there was general satisfaction with outcomes following gastrostomy insertion [30]. This was related to improved well-being and nutritional status of the child and for the parent reduced care demands and less concern about feeding and the delivery of medication [30]. In this study of the CDKL5 disorder, it is likely that some children may have had the gastrostomy placement in order to facilitate the provision of a ketogenic diet [35], which might have helped control seizures and in turn ease emotional burden of care for the parents. Although primary caregiver's physical health was not adversely affected by gastrostomy placement in those with a child with cerebral palsy [36], increased demands or excessive weight gain of the child could be contributing to mothers' physical burden in our study. Thus some deterioration in caregiver physical health may accompany better emotional health, which has been a consequence of less mealtime stress and improved child well-being.

Secondly, while a slight improvement in physical wellbeing was demonstrated, respite care use did not have a positive impact on the mental health of the primary caregivers. Furthermore, families who had utilised these services were less satisfied with every aspect of their family quality of life compared with non-users. Despite contrasting findings from a systematic review [37], individual studies have shown that service utilisation either had no positive effect [29] or was negatively associated with carer's emotional health $[38,39]$. Our longitudinal study involving families with a child with Rett syndrome found that respite care use did not improve parental emotional health and was associated with worse physical health over two years of follow-up [40]. Furthermore, in other research increased family needs for support have been reported to be associated with poorer family quality of life [41]. Our findings might suggest that needs for respite care are not being met for some families and that the 
Table 5 Multivariate analysis for the BCFQOL Scale and coefficients ${ }^{\# 1}$

\begin{tabular}{|c|c|c|c|c|c|c|}
\hline Measure & & Family interaction & Parenting & $\begin{array}{l}\text { Emotional } \\
\text { wellbeing }\end{array}$ & $\begin{array}{l}\text { Physical/Material } \\
\text { wellbeing }\end{array}$ & $\begin{array}{l}\text { Disability-related } \\
\text { support }\end{array}$ \\
\hline \multirow[t]{3}{*}{ Feeding pattern } & Oral intake only & Reference & Reference & Reference & Reference & Reference \\
\hline & Combination use & -0.12 & -0.16 & 0.13 & -0.16 & 0.13 \\
\hline & Enteral nutrition only & 0.07 & 0.02 & -0.06 & -0.03 & -0.06 \\
\hline \multirow[t]{3}{*}{ Dietary concerns } & None & Reference & Reference & Reference & Reference & Reference \\
\hline & Occasional & -0.15 & -0.25 & -0.08 & -0.25 & -0.19 \\
\hline & Frequent or constant & -0.29 & -0.29 & 0.19 & -0.19 & -0.26 \\
\hline \multirow[t]{3}{*}{ Floor sitting } & No assistance & Reference & Reference & Reference & Reference & Reference \\
\hline & Some assistance & -0.02 & 0.05 & 0.42 & -0.10 & 0.17 \\
\hline & Maximal assistance/Unable & 0.17 & 0.16 & 0.19 & 0.14 & 0.20 \\
\hline \multirow[t]{3}{*}{ Taking 10 steps forward } & No assistance & Reference & Reference & Reference & Reference & Reference \\
\hline & Some assistance & 0.21 & -0.03 & 0.13 & 0.02 & 0.09 \\
\hline & Maximal assistance/Unable & 0.18 & 0.08 & 0.25 & 0.09 & 0.07 \\
\hline \multirow[t]{2}{*}{ Eye contact } & No & Reference & Reference & Reference & Reference & Reference \\
\hline & Yes & -0.16 & -0.18 & -0.19 & -0.12 & -0.05 \\
\hline \multirow[t]{3}{*}{ Communication } & No/Simple method & Reference & Reference & Reference & Reference & Reference \\
\hline & Complex gestures/vocalisation & -0.12 & -0.11 & $-0.33^{*}$ & -0.11 & -0.15 \\
\hline & Sign/Spoken language & -0.02 & -0.02 & -0.31 & 0.03 & 0.06 \\
\hline \multirow[t]{5}{*}{ Seizure frequency } & Absent & Reference & Reference & Reference & Reference & Reference \\
\hline & Yearly/Monthly & -0.28 & -0.06 & 0.40 & -0.15 & 0.26 \\
\hline & Weekly & -0.12 & 0.13 & $0.57^{*}$ & -0.05 & 0.26 \\
\hline & 1 to 5 times a day & -0.25 & -0.06 & 0.13 & -0.06 & 0.18 \\
\hline & At least 5 times a day & -0.07 & 0.14 & 0.53 & 0.06 & 0.35 \\
\hline \multirow[t]{4}{*}{ Sleep difficulties } & 1.00 to 1.50 ( $1^{\text {st }}$ quartile $)$ & Reference & Reference & Reference & Reference & Reference \\
\hline & 1.50 to 1.81 ( $2^{\text {nd }}$ quartile) & -0.10 & -0.08 & -0.28 & -0.09 & -0.28 \\
\hline & 1.81 to 2.17 ( $3^{\text {rd }}$ quartile) & -0.06 & -0.03 & -0.26 & 0.08 & -0.10 \\
\hline & 2.17 to 5.00 ( $4^{\text {th }}$ quartile) & -0.15 & -0.09 & -0.26 & -0.16 & -0.23 \\
\hline \multirow[t]{4}{*}{ Hospitali-sation rate } & 0 to 0.38 ( $1^{\text {st }}$ quartile) & Reference & Reference & Reference & Reference & Reference \\
\hline & 0.38 to 0.86 ( $2^{\text {nd }}$ quartile) & 0.14 & 0.07 & 0.09 & -0.01 & 0.21 \\
\hline & 0.86 to 2.14 ( $3^{\text {rd }}$ quartile) & -0.07 & -0.18 & -0.37 & -0.22 & 0.08 \\
\hline & 2.14 to 13.70 ( $4^{\text {th }}$ quartile) & 0.10 & 0.24 & 0.09 & 0.03 & 0.13 \\
\hline \multirow[t]{4}{*}{ Respite care } & None & Reference & Reference & Reference & Reference & Reference \\
\hline & Formal only & -0.29 & -0.18 & $-0.72^{* *}$ & -0.07 & -0.01 \\
\hline & Informal only & -0.06 & -0.20 & $-0.47^{* *}$ & -0.23 & -0.28 \\
\hline & Both formal and informal & $-0.39^{* *}$ & -0.26 & -0.36 & $-0.32^{* *}$ & $-0.39 * *$ \\
\hline \multirow[t]{2}{*}{ Financial difficulty } & No & Reference & Reference & Reference & Reference & Reference \\
\hline & Yes & 0.11 & 0.04 & -0.27 & 0.03 & 0.02 \\
\hline
\end{tabular}

\#1 Adjusted for parent's age (a continuous variable), parent's qualification (a binary variable; university degree or lower), parent's work status, number of siblings, the first child, child's age (a continuous variable), child's country of birth (a binary variable; North America or others) and the scores of PCS and MCS (continuous variables)

${ }^{*} p<0.10{ }^{* *} p<0.05$

current framework for service delivery is not enhancing parental wellbeing or family quality of life. However, this issue warrants further investigation, possibly using either a longitudinal study design and/or qualitiative methods.
Thirdly, mothers in the youngest age group experienced the most impaired emotional health, possibly related to the recency of receiving their child's diagnosis, a time when they could be experiencing overwhelming fear and loneliness [42]. However, establishing a diagnosis 
is imperative so that families can clarify their child's expected life course and gain disease-specific family support [43]. Parents of a child with intellectual disability reported their wellbeing as better when the child had received a definite diagnosis [17]. As the CDKL5 gene testing has become available over the last decade, some of older primary caregivers in this study might have felt greatly relieved when their adult child eventually received a clear diagnosis. That said even in the general population mental health scores do improve with age [20], but for those with a CDKL5-affected child, we showed that the mean MCS score was consistently poorer across all age groups.

Lastly, family quality of life was strongly mediated by primary caregiver's health status. The mother's report of family quality of life has been assessed in most studies [16], and mothers under stress are more likely to consider their family quality of life as poorer [15]. In our study, adjustment for the SF-12v2 scores indicated that child's sleep difficulty had less impact on the whole family than on the primary carer. Controlling for maternal health would be of use to verify pathways from potential predictors to family quality of life.

We were surprised that there were no strong associations between the frequency of seizures or the attainment of gross motor and communication skills and family outcomes, given the major burden of epilepsy and the severe physical and intellectual impairment in the CDKL5 disorder. Previous studies on the effect of seizure control on maternal mental health have had mixed findings although mostly undertaken in children who were otherwise healthy [44-48]. The marked intellectual disability and various comorbidities among children with the CDKL5 disorder may lessen the influence of seizures alone on the primary caregiver's health.

Finally, we did not find any association between the rate of hospitalisations and primary caregiver health or family quality of life. In our study, all hospitalisations experienced by the child with the CDKL5 disorder over the life course were included, whereas the outcomes related to current status. We therefore may not be able to observe any true relationships.

Our study has several limitations. First, we used a cross-sectional study design and because of the absence of longitudinal data we were restricted to the reporting of associations rather than causal relationships. Secondly, despite the growth of our international database, the ICDD is not a population-based study. Hence, while remarkable heterogeneity in the carer's physical health and family quality of life were found according to child's country of birth, it might be explained by the uneven distribution of participants as well as voluntary participation. Thirdly, we did not account for the child's age when a diagnosis was made. Time intervals from when parents first had concerns about their child to the age at diagnosis and from the age at diagnosis to the present might influence the primary caregiver's emotional wellbeing. Fourthly, family quality of life was reported solely by the primary caregiver although adjustment for the SF-12v2 scores would have helped to counteract any confounding effect.

\section{Conclusions}

Despite its shortcomings, we believe that this study provides important insights into primary caregiver's wellbeing and family quality of life among families with a child with a severe genetic disorder, previously difficult to study because of its rarity. Child's feeding methods and sleep difficulties, and experiences of financial hardship were associated with primary caregiver's wellbeing, whereas use of respite care services was the principal correlate with family quality of life after controlling for the carer's health among families with a child with the CDKL5 disorder included in this study. To date, minimal attention has been given to families with a child living with a rare genetic disorder. Although we still acknowledge the need for further longitudinal investigation, the current research has only been possible for the CDKL5 disorder through the implementation and development of a worldwide database.

\begin{abstract}
Abbreviations
aa: Amino acid; BCFQOL: Beach center family quality of life; CDKL5: Cyclindependent kinase-like 5; ICDD: International CDKL5 disorder database; IFCR: International foundation for CDKL5 research; MCS: Mental component summary; PCS: Physical component summary; RTT: Rett syndrome; SDSC: Sleep disturbance scale for children; SF-12v2: Short form 12 health survey version 2
\end{abstract}

\section{Acknowledgements}

We would like to express our deep gratitude to all the families who have participated in the International CDKL5 disorder Database. We would also like to thank the International Foundation for CDKL5 Research for funding and ongoing support. We acknowledge the work of Dr. Stephanie Fehr and the Western Sydney Genetics Program at the Children's Hospital at Westmead, Sydney for interpretation of genetic reports.

\section{Funding}

This study as well as management of the International CDKL5 Disorder Database was supported by the International Foundation for CDKL5 Research.

\section{Availability of data and materials}

The datasets analysed during the current study are not publicly available but may be available from the corresponding author on reasonable request following an application to and with approval from the local ethics committee.

\section{Authors' contribution}

This study was conceptualised by HL and JD both of whom participated in the organisation and execution of the study including drafting and reviewing of the manuscript. YM participated with $\mathrm{HL}$ and JD in the study design and organisation. YM and KW designed and executed the statistical analysis. All authors contributed to the drafting and critique of the manuscript and read and approved the final manuscript. 


\section{Consent for publication}

Not applicable.

\section{Ethics approval and consent to participate}

Ethical approval for the study was obtained from the Ethics Committee of the University of Western Australia. Consent was obtained from all participants after information on the International CDKL5 Disorder Database was fully provided by the staff member.

\section{Author details}

'Telethon Kids Institute, The University of Western Australia, 100 Roberts Road, Subiaco, 6008 Perth, Western Australia. ${ }^{2}$ School of Physiotherapy and Exercise Science, Curtin University, Perth, Western Australia.

Received: 23 August 2016 Accepted: 27 December 2016 Published online: 19 January 2017

\section{References}

1. Weaving L, Christodoulou J, Williamson S, Friend K, McKenzie O, Archer H, et al. Mutations of CDKL5 cause a severe neurodevelopmental disorder with infantile spasms and mental retardation. Am J Hum Genet. 2004;75:1079-93.

2. Kalsheuer V, Tao J, Donnelly A, Hollway G, Schwinger E, Kubart S, et al. Disruption of the serine/threonine kinase 9 gene causes Dever $\mathrm{X}$-linked infantile spasms and mental retardation. Am J Hum Genet. 2003;72:1401-11.

3. Evans J, Archer H, Colley J, Ravn K, Nielsen J, Kerr A, et al. Early onset seizures and Rett-like features associated with mutations in CDKL5. Eur J Hum Genet. 2005;13:1113-20.

4. Fehr S, Wilson M, Downs J, Williams S, Murgia A, Sartori S, et al. The CDKL5 disorder is an independent clinical entity associated with early-onset encephalopathy. Eur J Hum Genet. 2013;21:266-73.

5. Guerrini R, Parrini E. Epilepsy in Rett syndrome, and CDKL5- and FOXG1gene-related encephalopathies. Epilepsia. 2012;53(12):2067-78.

6. Mangatt M, Wong K, Anderson B, Epstein A, Hodgetts S, Leonard H, et al. Prevalence and onset of comorbidities in the CDKL5 disorder differ from Rett syndrome. Orphanet J Rare Dis. 2016;11:39.

7. Fehr S, Wong K, Chin R, Williams S, de Klerk N, Forbes D, et al. Seizure variabiles and their relationship to genotype and functional abilities in the CDKL5 disorder. Neurology. 2016:87:2206-13.

8. Fehr S, Leonard H, Ho G, Williams S, Klerk N, Forbes D, et al. There is variability in the attainment of developmental milestones in the CDKL5 disorder. J Neurodev Disord. 2015;7:2.

9. Totsika V, Hastings R, Emerson E, Lancaster G, Berridge D. A populationbased investigation of behavioural and emotional problems and maternal mental stress: associations with autism spectrum disorder and intellectual disability. J Child Psychol Psychiatry. 2011;52(1):91-9.

10. Zablotsky B, Bradshaw C, Stuart E. The association between mental health, stress, and coping supports in mothers of children with autism spectrum disorders. J Autism Dev Disord. 2013;43:1380-93.

11. Bourke J, Ricciardo B, Bebbington A, Aiberti K, Jacoby P, Dyke P, et al. Physical and mental health in mothers of children with Down syndrome. J Pediatr. 2008;153:320-6.

12. Laurvick C, Msall M, Silburn S, Bower C, de Klerk N, Leonard H. Physical and mental health of mothers caring for a child with Rett syndrome. Pediatrics. 2006;118:e1152-64.

13. Cianfaglione R, Hastings R, Felce D, Clarke A, Kerr M. Psychological wellbeing of mothers and siblings in families of girls and women with Rett syndrome. J Autism Dev Disord. 2015;45:2939-46.

14. Trrdik T, Mason D, Dent K, Thornton L, Hornton S, Viskochil D, et al. Stress and coping in parents of children with Prader-Willi syndrome: assessment of the impact of a structured plan of care. Am J Med Genet. 2014;167A:974-82.

15. Gardiner E, larocci G. Unhappy (and happy) in their own way: a developmental psychopathology perspective on quality of life for families living with developmental disability with and without autism. Res Dev Disabil. 2012;33:2177-92.

16. Zuna N, Brown I, Brown R. Family quality of life in intellectual and developmental disabilities: a support-based framework. Int J Public Health. 2014;6(2):161-84.

17. Tibben A. Obtaining a genetic diagnosis in a child with disability: impact on parental quality of life. Clin Genet. 2016;89:258-66.
18. Louise S, Fyfe S, Bebbington A, Bahi-Buisson N, Anderson A, Pineda M, et al. InterRett, a model for international data collection in a rare genetic disorder. Res Autism Spect Dis. 2009;3:639-59.

19. Bruni O, Ottaviano S, Guidetti V, Romoli M, Innocenzi M, Cortesi F, et al. The sleep disturbance scale for children (SDSC) construction and validation of an instrument to evaluate sleep disturbances in childhood and adolescence. J Sleep Res. 1996;5:251-61.

20. Ware J, Kosinski M, Turner-Bowker D, Gandek B. How to score version 2 of the SF-12 health survey (with a supplement documenting version 1). Licoln, Rhode Island: QualityMetric Incorporated; 2004.

21. Samuel P, Rillotta F, Brown I. Review: the development of family quality of life concepts and measures. J Intellect Disabil Res. 2012;56(1):1-16.

22. Hoffman L, Marquis J, Poston D, Summers J, Turnbull A. Assessing family outcomes: psychometric evaluation of the beach center family quality of life scale. J Marriage Fam. 2006;68:1069-83.

23. Bourke-Taylor H, Pallant J, Law M, Howie L. Predicting mental health among mothers of school-aged children with developmental disabilities: the relative contribution of child, maternal and environmental factors. Res Dev Disabil. 2012:33:1732-40.

24. Lee J. Maternal stress, well-being, and impaired sleep in mothers of children with developmental disabilities: a liternature review. Res Dev Disabil. 2013;34:4255-73.

25. Chu J, Richdale A. Sleep quality and psychological wellbeing in mothers of children with developmental disabilities. Res Dev Disabil. 2009;30:1512-22.

26. Bourke-Taylor H, Pallant J, Law M, Howie L. Relationships between sleep disruptions, health and care responsibilities among mothers of school-aged children with disabilities. J Paediatr Child Health. 2013;49:775-82.

27. Hodge D, Hoffman C, Sweeney D, Riggs M. Relationship between children's sleep and mental health in mothers with and without autism. J Autism Dev Disord. 2013;43:956-63.

28. Wayte S, McCaughey E, Holley S, Annaz D, Hill C. Sleep problems in children with cerebral palsy and their relationship with maternal sleep and depression. Acta Paediatr. 2012;101:618-23.

29. Minnes P, Perry A, Weiss J. Predictors of distress and well-being in parents of young children with developmental delays and disabilities: the importance of parent perceptions. J Intellect Disabil Res. 2015;59(6):551-60.

30. Downs J, Wong K, Ravikumara M, Ellaway C, Elliot E, Christodoulou J, et al. Experience of gastrostomy using a quality care framework: the example of Rett syndrome. Medicine. 2014;93(28):e328.

31. Wilken $M$. The impact of child tube feeding on maternal emotional state and identity: a qualitative meta-analysis. J Pediatr Nurs. 2012;27:248-55.

32. Brotherton A, Abbott J, Aggett P. The impact of percutaneous endoscopic gastrostomy feeding in children; the parental perspective. Child Care Health Dev. 2007:33(5):539-46.

33. Mahant $\mathrm{S}$, Jovcevska $\mathrm{V}$, Cohen E. Decision-making around gastrostomyfeeding in children with neurologic disabilities. Pediatrics. 2011;127:e1471.

34. Nelson K, Lacombe-Duncan A, Cohen E, Nicholas D, Rosella L, Guttmann A, et al. Family experiences with feeding tubes in neurologic impairment: a systematic review. Pediatrics. 2015;136:e140-51.

35. Muller A, Helbig I, Jansen C, Bast T, Guerrini R, Jahn J, et al. Retrospectie evaluation of low long-term efficacy of antiepileptic drugs and ketogenic diet in 39 patients with CDKL5-related epilepsy. Eur J Paediatr Neurol. 2016;20:147-51.

36. Sullivan $P$, Juszczak E, Bachlet A, Lambert B, Vernon-Roberts A, Grant $H$, et al. Impact of gastrostomy tube feeding on the quality of life of cares of children with cerebral palsy. Dev Med Child Neurol. 2004;46:796-800.

37. Strunk J. Respite care for families of special needs children: a systematic review. J Dev Phys Disabil. 2010;22:615-30.

38. Sawyer M, Bittman M, Greca A, Crettenden A, Harchak T, Martin J. Time demands of caring for children with autism: what are the implications for maternal mental health? J Autism Dev Disord. 2010:40:620-8.

39. Carter A, Martinez-Pedraza F, Gray S. Stability and individual change in depressive symptoms among mothers raising young children with ASD: maternal and child correlates. J Clin Psychol. 2009;65(12):1270-80.

40. Urbanowicz A, Downs J, Bebbington A, Jacoby P, Girder S, Leonard H. Use of equipment and respite services and caregiver health among Australian families living with Rett syndrome. Res Autism Spectr Disord. 2011;5:722-32.

41. Boehm T, Carter E, Taylor J. Family quality of life during the transition to adulthood for individuals with intellectual difficulty and/or autism spectrum disorders. Am J Intellect Dev Disabil. 2015;120(5):395-411.

42. Glenn A. Using online health communication to manage chronic sorrow: mothers of children with rare diseases speak. J Pediatr Nurs. 2015;30:17-24. 
43. Moeschler J, Shevell M. Genetics Co. Comprehensive evaluation of the child with intellectual disability or global developmental delays. Pediatrics. 2014;134:e903-18.

44. Lv R, Wu L, Jin L, Lu Q, Wang M, Qu Y, et al. Depression, auxiety and quality of life in parents of children with epilepsy. Acta Neurol Scand. 2009;120:335-41.

45. Akay A, Kurul S, Ozek H, Cengizhan S, Emiroglu N, Ellidokuz H. Maternal reactions to a child with epilepsy: depression, anxiety, parental attitudes and family functions. Epilepsy Res. 2011;95:213-20.

46. Young $L$, Chengye J, Jiong Q. Factors affecting the quality of life in childhood epilepsy in China. Acta Neurol Scand. 2006;113:167-73.

47. Kerne V, Chapieski L. Adaptive functioning in pediatric epilepsy: contributions of seizure-related variables and parental anxiety. Epilepsy Behav. 2015;43:48-52.

48. Williams J, Steel C, Sharp G, DelosReyes E, Phillips T, Bates S, et al. Parental anxiety and quality of life in children with epilepsy. Epilepsy Behav. 2003;4:483-6.

Submit your next manuscript to BioMed Central and we will help you at every step:

- We accept pre-submission inquiries

- Our selector tool helps you to find the most relevant journal

- We provide round the clock customer support

- Convenient online submission

- Thorough peer review

- Inclusion in PubMed and all major indexing services

- Maximum visibility for your research

Submit your manuscript at www.biomedcentral.com/submit 